\title{
Making progress in genetic kin recognition among vertebrates
}

\author{
Jane L Hurst*1 and Robert J Beynon² \\ See research article http://www.biomedcentral.com/1471-2148/9/281
}

\begin{abstract}
A recent study in BMC Evolutionary Biology has shown that genetically similar individual ring-tailed lemurs are also more similar in their scent composition, suggesting a possible mechanism of kin recognition. Theoretical and experimental studies reveal challenges ahead in achieving a true systems-level understanding of this process and its outcomes.
\end{abstract}

Across a very broad taxonomic range animals frequently respond differentially to close kin, even if those kin were previously unfamiliar. Logically, this differentiation between individuals according to kinship requires welldefined mechanisms to allow recognition. And whereas animals may learn the cues of familiar individual kin during rearing, recognition of unfamiliar kin must really be recognition of genetic similarity - either to self or to other known kin. A challenge in this area lies in discovering the cues that animals use for genetic recognition of kin, and the genetic encoding of such cues. In many vertebrates, odors are key to the recognition process, and have been widely implicated as cues that allow genetic kin recognition in many species of fish, reptiles and mammals (Figure 1). However, vertebrate scents are generally complex, and there have been few attempts to identify the specific scent components used in kin recognition or their genetic basis.

\section{Gene-odor covariance}

In work published recently in BMC Evolutionary Biology, Boulet and colleagues [1] have advanced this field by

\footnotetext{
*Correspondence: jane.hurst@liv.ac.uk

'Mammalian Behaviour and Evolution Group, School of Veterinary Science, University of Liverpool, Leahurst Campus, Neston CH64 7TE, UK

2Proteomics and Functional Genomics Group, School of Veterinary Science, University of Liverpool, Crown Street, Liverpool L69 7ZJ, UK.
}

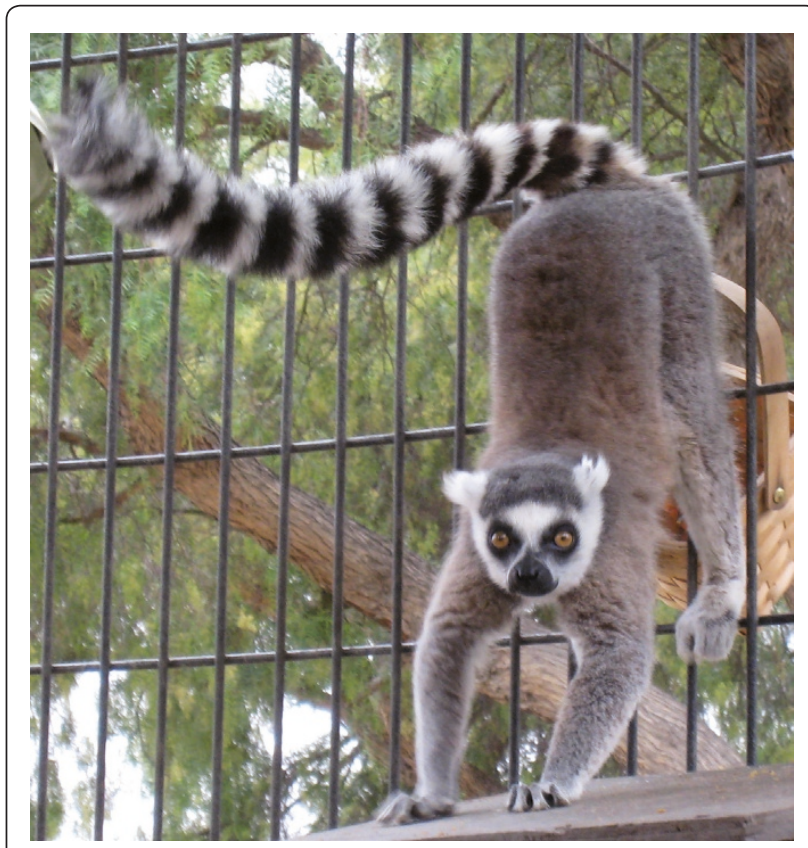

Figure 1. Ring-tailed Lemur (Lemur catta) using perianal glands for scent marking. (Photograph by Alex Dunkel/Visionholder).

demonstrating a significant correlation between genetic similarity (estimated from 11-14 microsatellite loci) in a captive population of ring-tailed lemurs (Lemur catta) and similarity of volatile chemicals in their genital gland secretions, as assessed by gas-chromatography massspectrometry. The genetic similarity of two individuals is thus manifest in the odor profile (sometimes referred to as an 'odortype'). Even more intriguing, although the genital glands of the two sexes are anatomically distinct (scrotal glands in the male, labial glands in the female), this covariance between genetic and chemical similarity is evident even between individuals of the opposite sex. While some components are expressed only by animals of one sex, more than half (about 170) were expressed by individuals of both sexes. To provide a simple estimate of chemical distance between a pair of individuals, Boulet et 
al. used the relative abundance of each of these shared compounds to calculate the Euclidean distance between the pair (derived from the Pythagorean theorem, this sums the pairwise difference, $\Delta$, in abundance of all 170 compounds, such that chemical distance $=\operatorname{SQRT}\left(\Delta_{1}{ }^{2}+\right.$ $\left.\Delta_{2}{ }^{2}+\Delta_{3}{ }^{2}+\ldots . \Delta_{170}{ }^{2}\right)$. While there was a broad spread of chemical distances between male-female dyads that had intermediate genetic distance, dyads with low genetic similarity had low chemical similarity whereas those with a high genetic similarity had a higher chemical similarity. This relationship is consistent with the hypothesis that odors from genital secretions can be used to assess genetic relatedness, and maybe close kinship. Of particular interest, these relationships were significant both within and between the sexes during the breeding season, but were much weaker or nonsignificant during the non-breeding season [1,2]. Odortype may be particularly important during the competitive breeding season to prevent inbreeding and/or to direct nepotistic behavior towards more closely related individuals.

However, this study is still only a first step in establishing whether such odor signals could offer a reliable means of recognizing kinship among ring-tailed lemurs and the genetic basis of the cues used. If lemurs used a measure of chemical distance based on all volatile compounds that are shared within or between the sexes, it would only be of very limited value in assessing kinship because of the considerable range in that measure between individuals of intermediate genetic relatedness. Although very closely related animals have similar odortypes, so do many individuals that are much less closely related. If odortype were used to avoid inbreeding, for example, the consequence would be exclusion as mates of many individuals that are not closely related, reducing choice without gaining any genetic benefit. It is likely, therefore, that animals use more specific markers within the odortype to distinguish close relatives reliably (Figure 2).

\section{Genetic and molecular mechanisms used to assess kinship}

There has been surprisingly little progress in establishing the genetic and molecular markers used to recognize kin through scents in vertebrates. In part, this may be due to the molecular complexity of vertebrate scents, which are the product not only of an individual's genes but also of hormonal and metabolic status, diet and microflora. For the past 30 years, the focus on genetic mechanisms underlying vertebrate kin recognition through odors has been on the major histocompatibility complex (MHC), which is often held to be the major genetic component apparently determining an individual's scent. Inbred laboratory mice have been a key model organism for manipulating

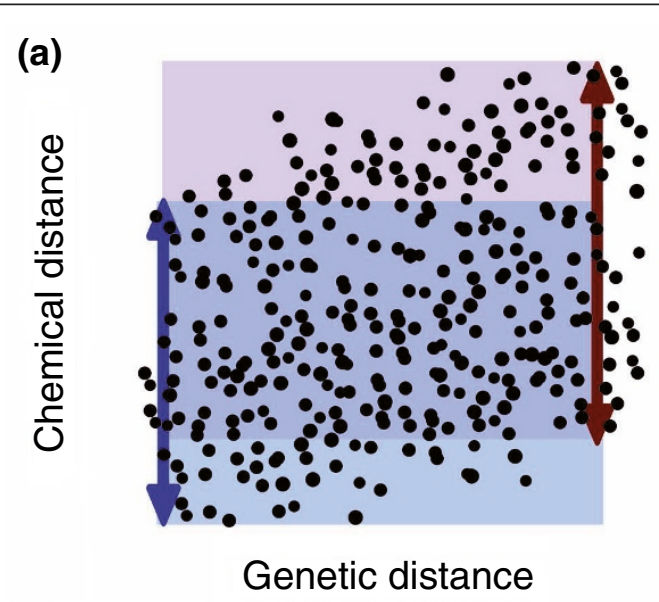

(b)

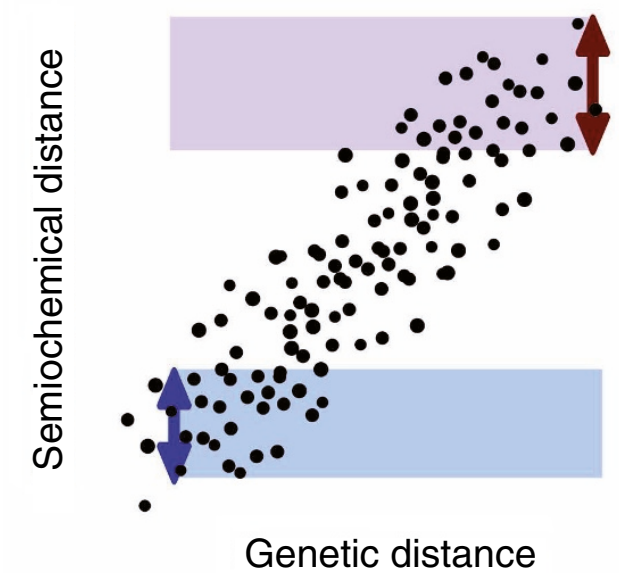

Figure 2. Model of gene-odor covariance for the reliable assessment of kinship. Chemical distance between pairs of animals based on all volatile compounds in a scent correlates with genetic distance (a), but variance will be high for any particular genetic distance because some compounds are likely to be strongly influenced by non-genetic factors such as current hormone levels and bacterial flora. Instead, selective assessment of specific semiochemicals within the scent that correlate strongly with genotype (b) will provide a much more reliable assessment of kinship.

MHC genes on a constant genetic background as proof that animals can detect MHC type through scent. As $\mathrm{MHC}$ is so highly polymorphic in natural populations, those that share the same MHC type (and MHC-based scent) are very likely to be closely related - MHC odors could be used as a marker of genetic relatedness. Yet, despite the precise genetic control offered by laboratory rodent strains, chemical analyses of volatile profiles have found correlations of some volatile components with MHC type but have not yet discovered consistent differences in compounds that are regulated by MHC type [3-6]. In reality, complex interactions are found with genetic background, 
microflora and diet, all of which alter the odor profile [3,5-7].

This plasticity of MHC-derived odortype creates a conundrum. To be useful in natural populations, kin markers must be stable and readily recognized against the variable genetic and environmental background of normal outbred animals. Our own studies of wildderived mice with normal genetic variation in seminatural populations provided clear evidence that wild mice do not use MHC to avoid inbreeding [8]. In fact, mice showed a very strong avoidance of inbreeding with those sharing another very highly polymorphic marker in mouse scent, the major urinary proteins (MUPs), which have a strong influence on an individual's scent profile regardless of other genetic and non-genetic variation [9].

Sharing of a single highly polymorphic marker, like MUP or MHC type, can provide a reliable indicator of relatedness because only close relatives are likely to inherit both of the same alleles at a particular locus (or both of the same haplotypes in the case of clusters of closely linked genes like MUP or MHC). However, this type of mechanism can only be partially effective for kin recognition. For any single locus, the number of alleles shared between two relatives is a matter of chance; even very close relatives such as full siblings are as likely to share no alleles as they are to share both alleles at a particular locus. Modeling alternative genetic mechanisms that could be used to discriminate full sibs from unrelated animals [10] reveals that reliance on a single genetic locus will either fail to identify many relatives (if the requirement is that both alleles are shared) or will mistake many unrelated animals as sibs (if sharing of any allele is used). Notwithstanding the theory, house mice do use sharing of MUP type, encoded by a single tightly linked cluster of genes, to avoid inbreeding [8]. This may be specific to house mice there are insufficient data to assess whether such simple recognition systems are widespread.

An alternative model is that instead of directly comparing the similarity of scents to self, imprinting on maternal scent encoded by several independent loci is employed to provide reliable recognition of all siblings and maternal half-sibs, because all offspring share with their mother one allele at every locus [10]. Laboratory cross-fostering studies in which newborn mouse pups were fostered onto a mother of different MHC type to their own have suggested that animals might imprint on the genotype of their mother and subsequently avoid 'inbreeding' with those sharing the foster mother's genotype rather than avoiding mates that match their own MHC type [11,12]. However, maternal imprinting does not require recognition of the mother's genotype for kin recognition; instead animals must be able to recognize the separate haplotypes carried by the mother when these are combined with other unknown haplotypes. This recognition task is likely to be considerably more difficult given the complex effects that MHC type has on odors, particularly as the odors of MHC heterozygotes are not an additive combination of the two homozygous profiles [3]. A key test would be whether mice (or other animals) can recognize the separate MHC haplotypes carried by a heterozygous animal when combined with other MHC haplotypes (for example, animals imprinted on the $\mathrm{MHC}^{\mathrm{bd}}$ haplotype must be able to recognize $\mathrm{MHC}^{\mathrm{bk}}$ or $\mathrm{MHC}^{\mathrm{dq}}$ ); they also need to be able to do this on the randomly assorting genetic background of outbred animals. Non-genetic maternal effects could also contribute to maternal imprinting for kin recognition. A recent study using inbred laboratory mice found that animals recognized non-genetic similarities in offspring from the same mother compared to those from another genetically identical female due to their shared maternal (in utero and postnatal) environment [13].

\section{The way forward}

The approach of relating genetic similarity to the global volatile profile of scent glands [1,2] is a step towards the systems biology of complex behaviors. Indeed, the application of global profiling methodologies to scents could be said to introduce the concept (but preferably not the term!) of 'semiomics'. As with many studies of this nature, the analyte mixtures are complex, and a major challenge is in unbundling the important semiochemicals from the entire volatile profile although Boulet and colleagues [1] refer to a 'semiochemical profile', it is likely that many of the constituent compounds will be 'silent' in kin recognition. An attractive way forward is to use the combined datasets to identify those chemicals that show the greatest correlation with relatedness, focusing on differences in relatedness that can be discriminated behaviorally. These chemicals then become the first candidates for testing with simple behavioral analyses. The candidates can be examined in 'kin-shifting' experiments such that when they are spiked into a distant sample, they elicit a response more 'akin' to a close relative. Indeed, similar experiments could be conducted using humans to establish the extent to which we too can discriminate our own kin based on genetically determined scents.

\section{Acknowledgements}

The development of these ideas was supported by research grants from BBSRC (S19816, BBC603897) and NERC (NEG018650).

Published: 17 February 2010 


\section{References}

1. Boulet M, Charpentier MJ, Drea CM: Decoding an olfactory mechanism of kin recognition and inbreeding avoidance in a primate. BMC Evol Bio/ 2009, 9:281.

2. Charpentier MJ, Boulet M, Drea CM: Smelling right: the scent of male lemurs advertises genetic quality and relatedness. Mol Ecol 2008, 17:3225-3233.

3. Willse A, Kwak J, Yamazaki K, Preti G, Wahl JH, Beauchamp GK: Individual odortypes: interaction of MHC and background genes. Immunogenetics 2006, 58:967-982

4. Novotny MV, Soini HA, Koyama S, Wiesler D, Bruce KE, Penn DJ: Chemical identification of MHC-influenced volatile compounds in mouse urine. I: Quantitative proportions of major chemosignals. J Chem Ecol 2007, 33:417-434.

5. Kwak J, Willse A, Matsumura K, Curran Opiekun M, Yi W, Preti G, Yamazaki K, Beauchamp GK: Genetically-based olfactory signatures persist despite dietary variation. PLoS One 2008, 3:e3591.

6. Zomer S, Dixon SJ, Xu Y, Jensen SP, Wang H, Lanyon CV, O'Donnell AG, Clare AS, Gosling LM, Penn DJ, Brereton RG: Consensus multivariate methods in gas chromatography mass spectrometry and denaturing gradient gel electrophoresis: MHC-congenic and other strains of mice can be classified according to the profiles of volatiles and microflora in their scent-marks. Analyst 2009, 134:114-123.

7. Rock F, Hadeler KP, Rammensee HG, Overath P: Quantitative analysis of mouse urine volatiles: in search of MHC-dependent differences. PLoS One 2007, 2:e429.
8. Sherborne AL, Thom MD, Paterson S, Jury F, Ollier WER, Stockley P, Beynon RJ, Hurst JL: The genetic basis of inbreeding avoidance in house mice. Curr Biol 2007, 17:2061-2066.

9. Cheetham SA, Thom MD, Jury F, Ollier WER, Beynon RJ, Hurst JL: The genetic basis of individual recognition signals in the mouse. Curr Biol 2007, 17:1771-1777.

10. Paterson S, Hurst JL: How effective is recognition of siblings on the basis of genotype? J Evol Biol 2009, 22:1875-1881.

11. Yamazaki K, Beauchamp GK, Kupniewski D, Bard J, Thomas L, Boyse EA Familial imprinting determines $\mathrm{H}-2$ selective mating preferences. Science 1988, 240:1331-1332

12. Penn D, Potts W: MHC-disassortative mating preferences reversed by crossfostering. Proc Biol Sci 1998, 265:1299-1306

13. Nakamura K, Kikusui T, Takeuchi Y, Mori Y: Influences of pre- and postnatal early life environments on the inhibitory properties of familiar urine odors in male mouse aggression. Chem Senses 2008, 33:541-551.

doi:10.1186/jbiol221

Cite this article as: Hurst JL and Beynon RJ. Making progress in genetic kin recognition among vertebrates. Journal of Biology 2010, 9:13. 